\title{
ÍNDICES DE CONFORT APLICADOS AL BALNEARIO DE MONTE HERMOSO, ARGENTINA
}

\author{
María A. Huamantinco Cisneros y M. Cintia Piccolo \\ CONICET- Instituto Argentino de Oceanografía \\ Departamento de Geografía y Turismo, Universidad Nacional del Sur (Bahía Blanca, Argentina)
}

\section{RESUMEN}

La planificación de las actividades de una ciudad y el bienestar de su población requieren del conocimiento de sus condiciones biometeorológicas, sobretodo en áreas turísticas. El área de estudio, balneario de Monte Hermoso, es el centro turístico con mayor crecimiento y expansión del suroeste bonaerense. El objetivo de la presente investigación es determinar y comparar el confort de Monte Hermoso durante la época estival de los años 2008 y 2009 a partir de la aplicación de tres índices de confort. Se utilizaron datos meteorológicos diarios obtenidos de la estación ubicada en la playa del balneario. Los parámetros considerados fueron temperatura, humedad y velocidad del viento a la hora de la máxima temperatura (13 horas). Las situaciones de confort y desconfort identificadas en cada uno de los índices fueron analizadas mediante gráficos de frecuencia. Los meses de enero y febrero son los que registran la mayor cantidad de días con desconfort climático. El mes de marzo se perfila como óptimo para el desarrollo de actividades recreativas y de esparcimiento. El periodo estival de 2009 fue el más desconfortable de los dos años analizados.

Palabras clave: confort climático, índices de confort, Monte Hermoso.

\begin{abstract}
The knowledge of biometeorological conditions is necessary in order to plan the urban activities and to protect its population. Monte Hermoso coastal city is a tourist city located in the Southwest of Buenos Aires Province. The objective of this investigation is determine and compare the climate comfort indexes applied to Monte Hermoso during the summer of 2008 and 2009. Three indexes of
\end{abstract}


comfort were analized. Meteorological data of temperature, humidity and wind velocity was used. This data was obtained from a meteorological station located on the beach of Monte Hermoso. January and February registered a great quantity of days of descomfort. March is the optimal month to organize recreational activities. Summer of 2009 was the most uncomfortable season of both years.

Key words: climate comfort, comfort indexes, Monte Hermoso.

\section{INTRODUCCIÓN}

El estudio de las condiciones biometeorológicas de una ciudad contribuye con la planificación de sus actividades y el bienestar de su población. Su consideración permite identificar aquellos momentos en los que el ser humano experimenta sensaciones de confort climático. Este aspecto resulta de importancia en espacios concurridos o habitados, como son los balnearios turísticos.

El clima y el tiempo son factores limitantes para el desarrollo de la actividad turística y, por otro lado, dominantes en la demanda turística como la elección del destino o las actividades que se van a realizar, ya que la participación del turista decrecerá si el desconfort e insatisfacción aumenta. Se vinculan con la salud, al considerar aquellas áreas climáticamente estresantes para viajes y actividades a grupos riesgosos (ancianos, enfermos y niños) (de Freitas, 2001; Matzarakis, 2006).

Las definiciones elaboradas, en distintos países, con respecto a la temperatura óptima para el ser humano demuestran que la sensación de bienestar climático es universal (Brazol, 1951). La relación existente entre el clima y el hombre ha sido motivo de diversas investigaciones desde fines del siglo XIX. Se incluyen, entre ellas, la confección de diagramas bioclimáticos o la formulación de índices de confort.

Otros trabajos sobre el confort climático se orientan al estudio del comportamiento de la población, frente a determinadas condiciones térmicas y de confort y los usos que hacen de los espacios urbanos al aire libre (Nikolopoulou et al., 2001) o al análisis del confort en espacios abiertos o cerrados, desde sus distintas definiciones (Höppe, 2002). También se han centrado en la cuantificación del stress térmico mediante la aplicación de diferentes índices (Mayer y Höppe, 1987) o al desarrollo de nuevos índices y modelos climáticos (Nikolopoulou et al., 2003; Stathopoulos et al., 2004).

Desde el punto de vista del turismo, se utilizan índices bioclimáticos que combinan diferentes elementos del ambiente y que permiten delimitar las respuestas del organismo frente a ellos. Entre los más seguros y simples, Besancenot (1991) menciona el complejo termo-anemométrico, que combina temperatura y viento, y el complejo termo-higrométrico, que asocia temperatura y humedad. Estos índices de confort, también, han sido utilizados junto con otros parámetros con el fin de evaluar la aptitud climática de destinos turísticos como Cataluña en España (Gómez Martín 2002, 2004a, b) y Jardines del Rey en Cuba (Batista Tamayo y Matos Pupo, 2004). 
En Argentina se han realizado estudios sobre confort climático. Algunos efectuaron un reconocimiento bioclimático del país e identificaron la cantidad de meses con bienestar climático (Brazol, 1954) o se orientaron a las clasificaciones bioclimáticas y su distribución geográfica (Hoffmann y Medina, 1971), mientras que otros centraron sus análisis en sectores de la costa del sudoeste bonaerense (Marini y Piccolo, 2000) o en ciudades como Bahía Blanca (Capelli de Steffens et al., 2005) y Monte Hermoso (Varela, 1982; Huamantinco Cisneros y Piccolo, 2008).

El área de estudio, balneario de Monte Hermoso, se ubica en el suroeste

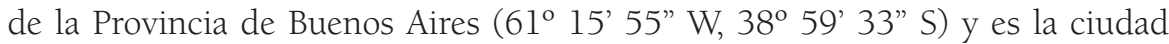
cabecera del partido del mismo nombre. Su costa posee una dirección E-W, con una extensión de $32 \mathrm{~km}$ entre Punta Sauce, a $14 \mathrm{~km}$ al Este del balneario, y punta Pehuen Co al Oeste (Figura 1). El perfil característico de su playa está compuesto por barras y canales, además de la presencia de médanos frontales. En algunos sectores, estos médanos han sido reemplazados por construcciones y obras de infraestructura debido a una urbanización no planificada (Fernández et al., 2006). El área urbana, con una extensión de 186 ha, se dispone de manera longitudinal, siguiendo la zona costera (Del Pozo, 2001). El ancho aproximado de la playa es de $260 \mathrm{~m}$, mientras que en algunos sectores céntricos de la ciudad ronda los $160 \mathrm{~m}$.

Figura 1. Ubicación del área de estudio

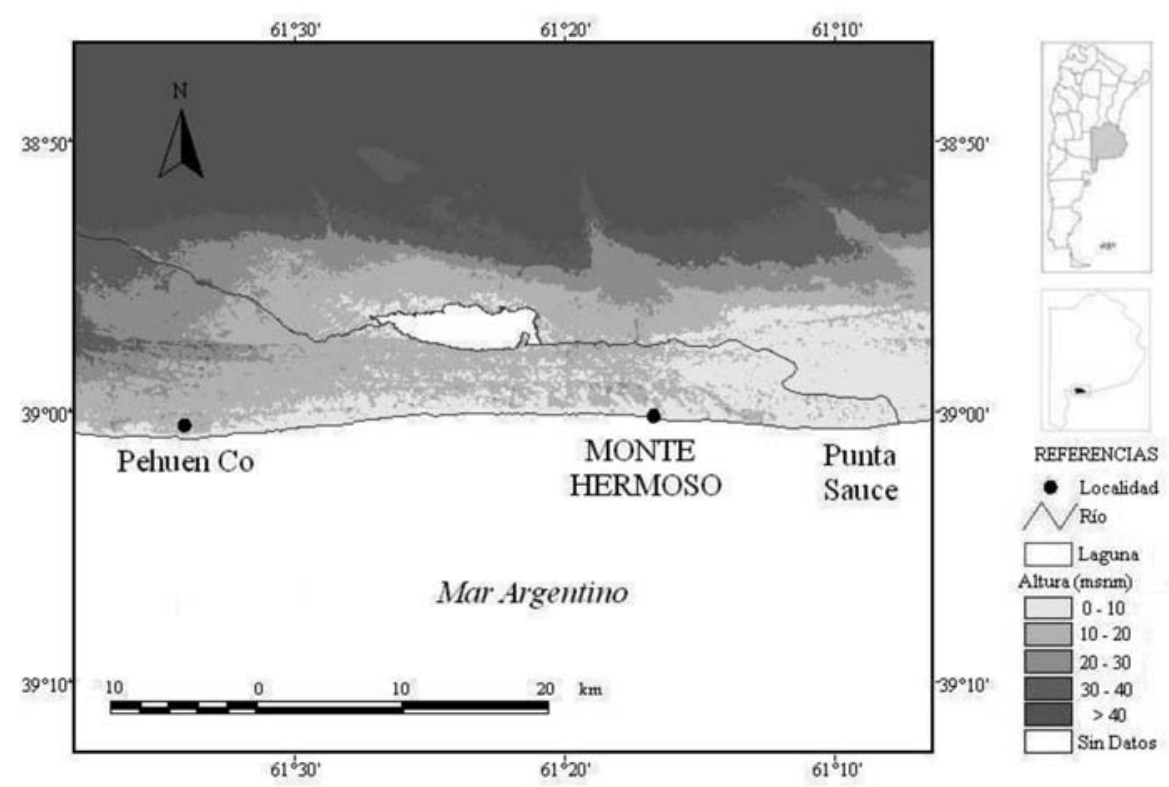

Investigaciones geográficas, $n^{\circ} 52$, pp. 201 - 214 
Desde el punto de vista demográfico, Monte Hermoso ha experimentado un destacado crecimiento desde 1983, año de creación del Partido homónimo. El censo de 1980 indicó un núcleo estable de 3100 personas. Este número registró un aumento, luego del censo de 1991, con una población permanente de 3605 habitantes (Ercolani y Visciarelli, 2003; Vaquero et al., 2007). Los datos censales del año 2001 indicaron una población estable de 5394 habitantes (INDEC, 2001).

Se destaca el turismo como principal actividad económica de la localidad (Vaquero y Pascale, 2003). Este rasgo ha favorecido el mantenimiento de la población estable y, con ello, su evolución de balneario a centro turístico, caracterizado por un uso intensivo del espacio litoral. Se convierte así en el centro turístico de mayor crecimiento y expansión del Suroeste Bonaerense (Vaquero et al., 2007).

En Monte Hermoso el flujo de personas hacia la costa se evidencia por la gran cantidad de vehículos que ingresan y circulan por la zona urbana en la época estival. En la temporada 1998/99 ingresaron a Monte Hermoso 122.113 vehículos (Del Pozo, 2001). Según datos suministrados por la Secretaría de Turismo de la Municipalidad de Monte Hermoso (2009), ingresaron 206.376 vehículos durante la temporada 2008/2009. Este dato demuestra el creciente flujo hacia este centro turístico.

En su estudio sobre Monte Hermoso, Varela (1982) confeccionó cartas bioclimáticas para los meses de verano de los años 1981 y 1982. Para ello, utilizó el diagrama de Olgyay, sobre el cual graficó los valores medios horarios mensuales de temperatura $\left({ }^{\circ} \mathrm{C}\right.$ ) y humedad relativa (\%). El mes de diciembre de 1980-1981 mostró un patrón de bienestar climático con una humedad relativa superior al 60\% y una mayor cantidad de horas de confort (14) durante el día. El análisis del mes de enero de 1981-1982 determinó confortabilidad durante la mañana, en las últimas horas de la tarde y en las primeras horas de la noche. La humedad relativa se ubicó entre el 62 y $78 \%$, con temperaturas medias que oscilaron entre los 19 y $24^{\circ} \mathrm{C}$. El desconfort fue percibido por la noche-madrugada (fresco y húmedo, $19-17^{\circ} \mathrm{C}$; 78-84\%). El mes de febrero de 1981-1982 presentó una sensación de confort durante gran parte del día, exceptuando las horas de la madrugada, con una humedad relativa superior al $55 \%$. Las temperaturas medias se ubicaron entre los 19 y $25^{\circ} \mathrm{C}$.

En el estudio realizado por Huamantinco Cisneros y Piccolo (2008) en el mismo núcleo urbano, se aplicó el índice Humidex y se confeccionaron diagramas bioclimáticos para los días con las máximas temperaturas mensuales de la temporada estival 2007-2008, con el objeto de determinar el confort térmico de Monte Hermoso. Los resultados analizados a partir del índice mencionado indicaron situaciones de desconfort en los días más calurosos durante 14 hs aproximadamente. La acción del viento no alcanzó a calmar la sensación de desconfort. El mismo se caracterizó por ser principalmente del cuadrante Norte, impidiendo el ingreso de la brisa de mar, que se refleja en un ascenso de la temperatura y dis- 
minución de la humedad. Si bien los días analizados correspondieron a los más cálidos de cada mes, al comparar los diagramas bioclimáticos, enero presentó mayor cantidad de horas de desconfort.

Los estudios realizados en 1982 (Varela) y 2008 (Huamantinco Cisneros y Piccolo) presentan un análisis parcial del confort térmico del balneario. En el primero, los valores medios horarios mensuales permitieron identificar las horas más favorables durante el mes. Para el segundo estudio, el análisis del confort en los días más cálidos advirtió sobre la cantidad de horas de malestar térmico. A fin de identificar la cantidad de días de desconfort en verano, el objetivo de la presente investigación es determinar y comparar el confort del balneario de Monte Hermoso durante la época estival de los años 2008 y 2009 a partir de la aplicación de tres índices de confort. Para ello, se consideran los valores medios diarios de la hora de máxima temperatura de los periodos en cuestión.

\section{MÉTODO DE TRABAJO}

Se utilizaron los datos diarios de temperatura $\left({ }^{\circ} \mathrm{C}\right)$, humedad $(\%)$ y viento $(\mathrm{km} / \mathrm{h})$ correspondientes a los tres meses de la temporada estival 2008 y 2009 obtenidos de la estación meteorológica de Monte Hermoso, localizada en la playa del balneario. Se calculó el índice de confort correspondiente a las 13 hs, hora de la máxima temperatura. Para el cálculo de confort se aplicaron tres índices: el Termo-anemométrico de Siple y Passel (1945), el Termo-higrométrico de Thom (1959) y el Humidex (Capelli de Steffens et al., 2005). Los dos primeros índices continúan utilizándose, tal es el caso de los trabajos de Gómez Martín (2002, 2004 a, b) y Batista Tamayo y Matos Pupo (2004). La elección de los mismos se basa en la facilidad para calcularlos y en la disponibilidad de los datos. El índice Humidex es una innovación canadiense y uno de los más utilizados desde 1965.

El primero de ellos (1) determina el poder refrigerante del aire, donde evalúa la cantidad de calor descontada al cuerpo durante una unidad de tiempo y se expresa en $\mathrm{kcal} / \mathrm{m}^{2} / \mathrm{h}$. La fórmula es la siguiente:

$$
\mathrm{P}=\left(10 \mathrm{~V}^{0.5}+10.45-\mathrm{V}\right) *(33-\mathrm{T})
$$

donde $\mathrm{P}=$ poder refrigerante del aire; $\mathrm{V}=$ velocidad del viento $\left(\mathrm{m} \mathrm{s}^{-1}\right)$ y $\mathrm{T}=$ temperatura máxima diaria $\left({ }^{\circ} \mathrm{C}\right)$.

El límite mínimo para este índice es de $50 \mathrm{kcal} / \mathrm{m}^{2} / \mathrm{h}$. Por debajo de este valor la sensación de desconfort se acentúa peligrosamente. Esto se debe porque «cuando la temperatura exterior supera los $33^{\circ} \mathrm{C}$, temperatura habitual de la piel desnuda, el cuerpo es incapaz de ceder directamente al aire su exceso de calorías, ya que este aire es en adelante más cálido que él» (Besancenot, 1991: 36). Las condiciones óptimas de confort se encuentran entre 300 y $599 \mathrm{kcal} / \mathrm{m}^{2} / \mathrm{h}$. Al considerarlo desde el punto de vista turístico, el rango se amplía de 50 a $599 \mathrm{kcal} / \mathrm{m}^{2} / \mathrm{h}$. 
El Índice Termo-higrométrico (THI) indica la temperatura que siente el hombre en ${ }^{\circ} \mathrm{C}$, al considerar la temperatura máxima diaria y la humedad relativa del aire (2). El cálculo se realiza con la siguiente fórmula:

$$
\mathrm{THI}=\mathrm{T}-[0.55-(0.0055 * \mathrm{HR}) *(\mathrm{~T}-14.5)](2)
$$

donde $\mathrm{T}=$ temperatura máxima $\left({ }^{\circ} \mathrm{C}\right)$ y $\mathrm{HR}=$ humedad relativa (\%). La sensación de confort se hace presente entre los 15 y $20^{\circ} \mathrm{C}$ de THI. Aunque el ser humano puede tolerar, en verano, sensaciones de hasta $28,5^{\circ} \mathrm{C}$. Cuando el aire no está saturado de vapor de agua, una persona tiene mayores posibilidades de soportar el aumento de la temperatura del aire.

El Humidex (H) es un índice de calor que se expresa en grados (3). Su objetivo es, bajo determinadas condiciones de humedad y temperatura ambiente, indicar la temperatura efectiva que siente el ser humano (Capelli de Steffens et al., 2005). Su fórmula es la siguiente:

$$
\mathrm{H}=\mathrm{T}+5 / 9 *(e-10)(3)
$$

donde $\mathrm{T}$ es la temperatura del aire $\left({ }^{\circ} \mathrm{C}\right)$ y e es la presión de vapor de aire en $\mathrm{hPa}$ (Capelli de Steffens et al., 2005).

El rango de desconfort que corresponde a esta fórmula se muestra a continuación:

\begin{tabular}{|l|l|}
\hline $20-29^{\circ}$ & confortable \\
\hline $30-39^{\circ}$ & algún desconfort \\
\hline $40-45^{\circ}$ & gran desconfort, evitar los ejercicios físicos \\
\hline$>45^{\circ}$ & peligro \\
\hline$>54^{\circ}$ & inminente peligro de sufrir infarto al corazón \\
\hline
\end{tabular}

En cada uno de los índices se identificaron las situaciones de confort y desconfort en el periodo considerado. Ambas situaciones fueron analizadas mediante un análisis de frecuencia (\%). La variación en el total de días medidos se debe a que algunos parámetros, requeridos para el cálculo de los índices, no fueron registrados.

\section{RESULTADOS}

El periodo estival 2008 y 2009 del balneario Monte Hermoso se caracterizó por temperaturas máximas entre 30 y $39^{\circ} \mathrm{C}$, con una media mensual de $21^{\circ} \mathrm{C}$ para el periodo de estudio. Los vientos predominaron del cuadrante Norte, con una velocidad media de $6 \mathrm{~m} \mathrm{~s}^{-1}$. La humedad relativa media fue del 40\% (2008) y $62 \%$ (2009).

Durante el mes de enero de 2008, el índice P indicó un solo día de desconfort para la población (Tabla 1). El índice mostró un valor negativo $\left(-8,54 \mathrm{kcal} / \mathrm{m}^{2} / \mathrm{h}\right)$ 
que evidenció un malestar térmico por efectos del calor. Esto fue producto de una temperatura máxima de $33,3{ }^{\circ} \mathrm{C}$, asociada a una velocidad de viento de 5,6 $\mathrm{m} \mathrm{s}^{-1}$ proveniente del sector Norte. El resto del mes el índice P presentó condiciones de confort, con temperaturas entre los 18 y $30^{\circ} \mathrm{C}$. Esta sensación de bienestar estuvo asociada a vientos continentales del sector Norte, con velocidades de 3 a $8 \mathrm{~m} \mathrm{~s}^{-1} \mathrm{y}$ marinos de los sectores E, SSE, S y SW, que oscilaron entre los 9 y $18 \mathrm{~m} \mathrm{~s}^{-1}$.

Tabla 1. Cantidad de días de confort y desconfort en los meses de enero a marzo de 2008 y 2009

\begin{tabular}{|c|c|c|c|c|c|c|c|c|c|c|c|c|c|c|c|c|c|c|}
\hline \multirow{2}{*}{ Días } & \multicolumn{3}{|c|}{ Enero '08 } & \multicolumn{3}{|c|}{ Enero '09 } & \multicolumn{3}{|c|}{ Febrero '08 } & \multicolumn{3}{|c|}{ Febrero '09 } & \multicolumn{3}{|c|}{ Marzo ‘08 } & \multicolumn{3}{|c|}{ Marzo '09 } \\
\hline & $\mathrm{P}$ & THI & $\mathrm{H}$ & $\mathrm{P}$ & THI & $\mathrm{H}$ & $\mathrm{P}$ & THI & $\mathrm{H}$ & $\mathrm{P}$ & THI & $\mathrm{H}$ & $\mathrm{P}$ & THI & $\mathrm{H}$ & $\mathrm{P}$ & THI & $\mathrm{H}$ \\
\hline Confort & 27 & 15 & 9 & 18 & 11 & 9 & 25 & 9 & 9 & 14 & 11 & 12 & 31 & 20 & 18 & 25 & 16 & 15 \\
\hline Desconfort & 1 & 6 & 12 & 8 & 13 & 15 & 2 & 12 & 12 & 2 & 15 & 14 & . & 5 & 6 & 5 & 14 & 15 \\
\hline $\begin{array}{l}\text { Total días- } \\
\text { medición }\end{array}$ & 28 & 21 & 21 & 26 & 24 & 24 & 27 & 21 & 21 & 16 & 26 & 26 & 31 & 25 & 24 & 30 & 30 & 30 \\
\hline
\end{tabular}

El mes de enero de 2009 presentó 8 días de desconfort de un total de 26 registrados (Tabla 1). La sensación de malestar térmico percibida por la población se debió a temperaturas superiores a los $30^{\circ} \mathrm{C}$ y vientos que predominaron del cuadrante NNE, con velocidades que oscilaron entre los 5 y $8 \mathrm{~m} \mathrm{~s}^{-1}$. La sensación de confort se manifestó con temperaturas entre 19 y $27^{\circ} \mathrm{C}$ y vientos con velocidades entre los 3 y $19 \mathrm{~m} \mathrm{~s}^{-1}$.

En febrero de 2008 y 2009 la situación de confort dominó, prácticamente, durante todo el mes. Ambos periodos presentaron sólo dos días desconfortables (Tabla 1). Los mismos se caracterizaron por temperaturas superiores a los $30^{\circ} \mathrm{C}$, acompañadas por vientos del cuadrante Norte a $7 \mathrm{~m} \mathrm{~s}^{-1}$ o a la presencia de calmas. Las sensaciones de bienestar climático se evidenciaron con temperaturas entre los $20 \mathrm{y}$ $28^{\circ} \mathrm{C}$ y vientos del sector W, NW, SW y WSW, con velocidades entre los 5 y $15 \mathrm{~m} \mathrm{~s}^{-1}$.

A diferencia de los meses anteriores, marzo de 2008 presentó situación de confort todos los días (Tabla 1; Figura 2). En marzo de 2009, si bien predominó la sensación de confort, la población experimentó 5 días de desconfort (Tabla 1). Estos se caracterizaron por temperaturas superiores a los $30^{\circ} \mathrm{C}$ y vientos del sector Norte, con velocidades entre 4 y $8 \mathrm{~m} \mathrm{~s}^{-1}$.

Al aplicar el índice THI, enero de 2008 se identificó como confortable, presentando 6 días con desconfort de un total de 21 días registrados (Tabla 1). Al año siguiente, enero fue más desconfortable, al presentar 13 días de malestar climático de un total de 24 (Tabla 1). Las situaciones de desconfort fueron percibidas por la población con temperaturas superiores a los $29^{\circ} \mathrm{C}$ y porcentajes variables de humedad relativa. 
Figura 2. Índice termo-anemométrico y temperatura del mes de marzo de 2008

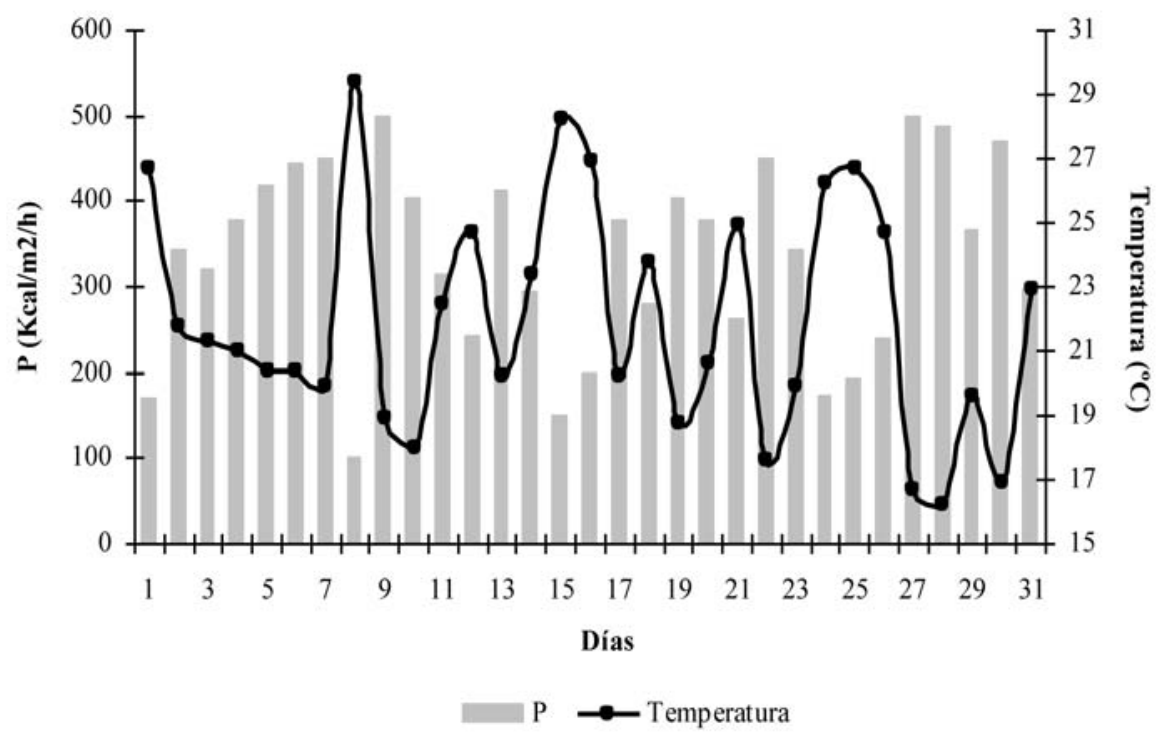

El mes de febrero de 2008 y 2009 se caracterizó por una situación de desconfort con 12 y 15 días, respectivamente (Tabla 1). Las temperaturas superaron los $30{ }^{\circ} \mathrm{C}$ y la humedad relativa osciló entre el 20 y 80\%. Los máximos valores del índice rondaron los $40^{\circ} \mathrm{C}$.

En el mes de marzo de 2008 y 2009, el índice THI indicó una predominancia en la sensación de confort (Tabla 1). En marzo de 2008, de un total de 25 días, 5 fueron de desconfort (Tabla 1). El día más desconfortable tuvo un valor de $33^{\circ} \mathrm{C}$. Durante el mes de marzo de 2009, 14 fueron los días de desconfort, que se expresó con valores entre los 29 y $36{ }^{\circ} \mathrm{C}$ (Figura 3; Tabla 1).

El índice H indicó, para el mes de enero de 2008 y 2009, una preponderancia de situaciones de desconfort. En el primero, con 12 días de «algún desconfort» $\left(30-39^{\circ}\right)$ de un total de 21 registrados. Mientras que el segundo contabilizó 15 días de malestar térmico, de los cuales 13 representaron situación de «algún desconfort» y 2 de «gran desconfort» $\left(40-45^{\circ}\right.$ ) (Tabla 1 ).

En el mes de febrero, el índice H evidenció un predominio de la sensación de confort tanto en 2008 como en 2009 (Tabla 1). De un total de 21 días registrados en 2008, 12 representaron situaciones de «algún desconfort» (10 días) y «gran desconfort» (2 días). En 2009, el mes de febrero presentó 14 días de desconfort de un total de 26 (Tabla 1).

El índice H señaló al mes de marzo de 2008 como confortable, con 18 días de un total de 24 (Tabla 1). La sensación de desconfort fue percibida el resto de 
los días, con valores entre 30 y $39^{\circ}$. Durante el mes de marzo de 2009, las sensaciones de confort y desconfort contabilizaron 15 días cada una (Tabla 1). La población percibió el bienestar térmico con temperaturas de 18 a $25^{\circ} \mathrm{C}$ y «algún desconfort» entre los 23 y $33^{\circ} \mathrm{C}$ (Figura 4).

Figura 3. Índice termo-higrométrico y temperatura del mes de marzo de 2009

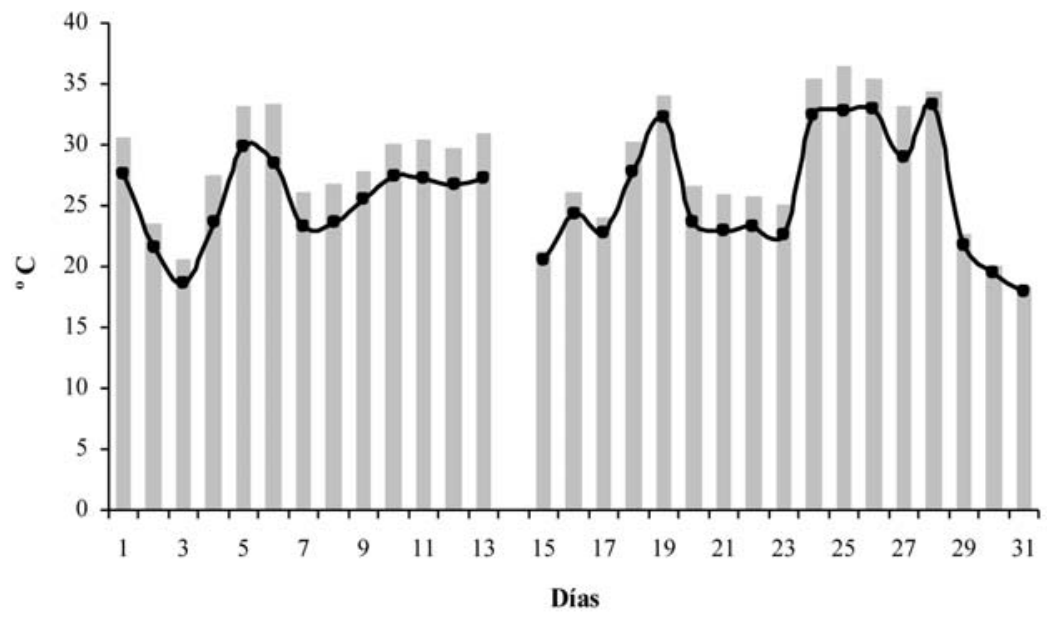

$\longrightarrow$ THI $\rightarrow$ Temperatura

Figura 4. Índice Humidex y temperatura del mes de marzo de 2009

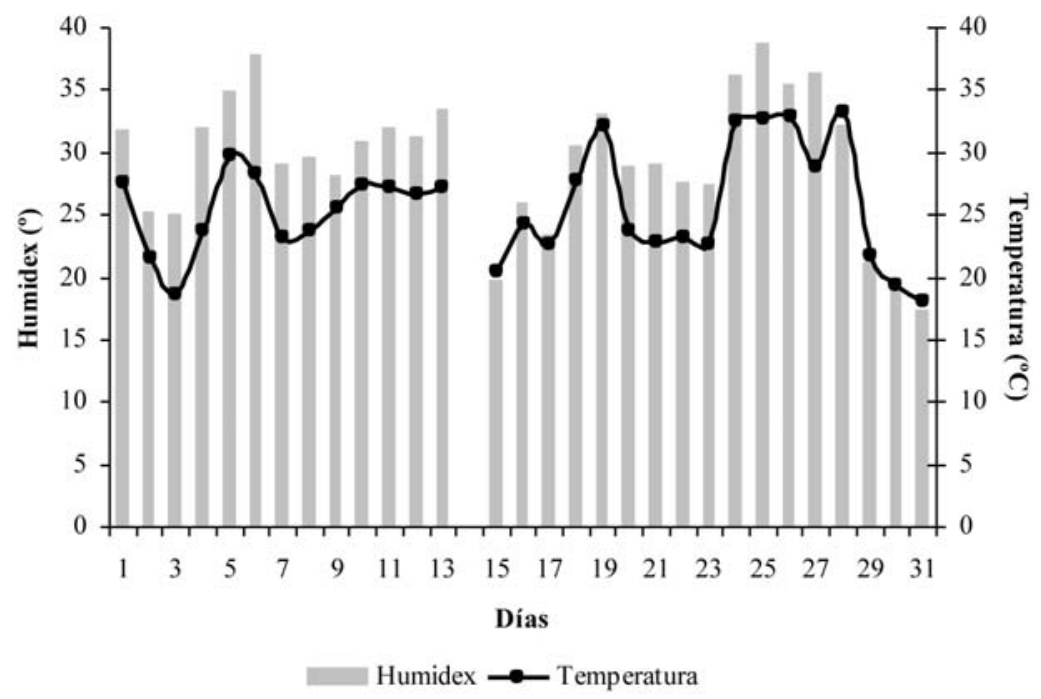


Al analizar la frecuencia del índice termo-anemométrico (P) se observa que todo el periodo considerado para el 2008 presentó sensaciones de confort climático en un 90 y 100\% (Figura 5). Los episodios de desconfort se observaron en enero y febrero, con el 3,6 y 7,4\% de los casos, respectivamente (Figura 5a). En el periodo 2009 se evidenció un predominio en la sensación de confort. La misma se expresó, para el total de casos considerados, en un 69,2, 87,5 y $83,3 \%$ para los meses de enero, febrero y marzo, respectivamente. De acuerdo a este índice, la sensación de desconfort fue notoria durante el mes de enero, con $30,8 \%$, mientras que febrero fue el mes con menor frecuencia, con el $12,5 \%$ de los casos (Figura 5b).

Figura 5. Frecuencia del índice termo-anemométrico para los años: a) 2008 y b) 2009
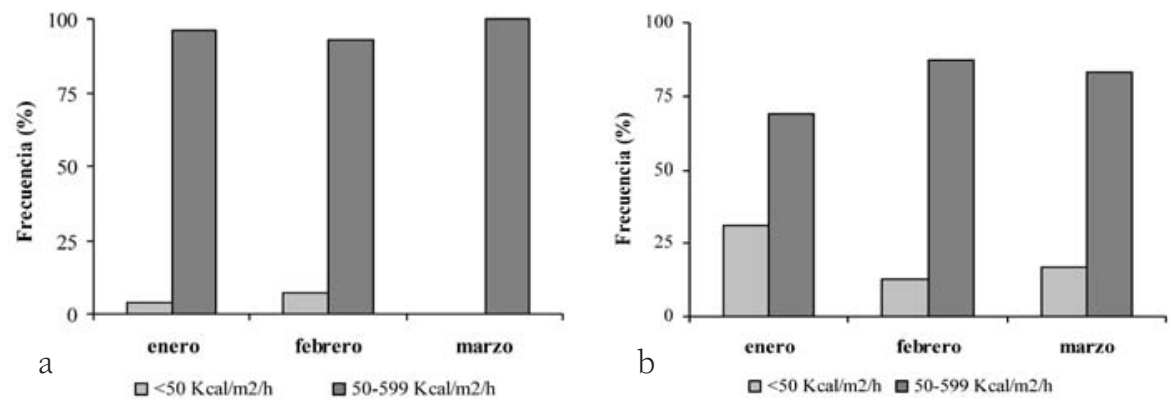

La frecuencia del índice termo-higrométrico (THI) presentó, para el periodo 2008, condiciones de confort para la población (Figura 6). Las mismas se evidenciaron con un 71 y $80 \%$ del total de los casos para los meses de enero y marzo, respectivamente. Se destacó el mes de febrero, por ser el más desconfortable con un 80\% (Figura 6a). El año 2009 indicó sensaciones de desconfort para los meses de enero $(54,2 \%)$ y febrero $(57,7 \%)$. Marzo, en cambio, presentó menor cantidad de casos con desconfort (46,7\%) (Figura 6b).

El índice Humidex $(\mathrm{H})$ señala un $57 \%$ de desconfort en los meses de enero y febrero para el periodo estival 2008. El mes de marzo, en cambio, fue el más confortable, con el 75\% (Figura 7a). En 2009, enero y febrero continuaron siendo los meses más desconfortables. Entre los rangos de «algún desconfort»y «gran desconfort», el mes de enero evidenció un $62,5 \%$ y febrero un 53,8\% del total de los casos (Figura 7b).

$\mathrm{Al}$ aplicar tres índices diferentes se comprobó que éstos no presentaron la misma cantidad de días de confort y desconfort durante el periodo estudiado. El motivo de esta variación es la consideración de distintos parámetros en su cálculo. Estos índices brindan una aproximación a la situación de confort percibida por la población. Así, 
Figura 6. Frecuencia del índice termo-higrométrico para los años: a) 2008 y b) 2009


Figura 7. Frecuencia del índice Humidex para los años: a) 2008 y b) 2009

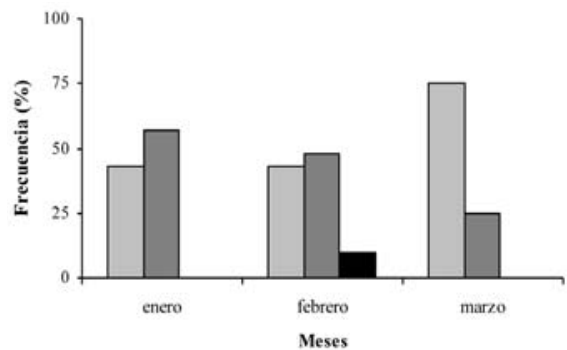

a

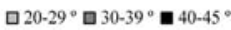

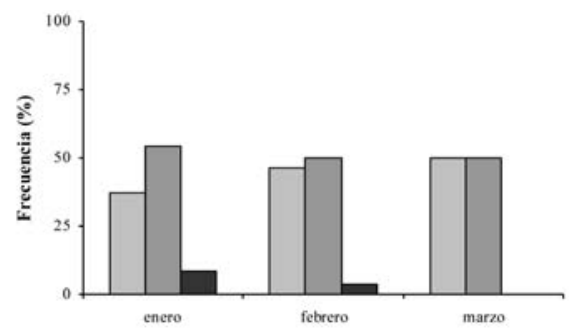

$\mathrm{b}$

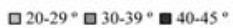

los índices THI y H demostraron una mayor cantidad de días de desconfort. Un rasgo que se debe señalar es que sus unidades son las que la población puede relacionar a la sensación de confort más rápidamente, al encontrarse expresados en ${ }^{\circ} \mathrm{C}$ y grados ${ }^{\circ}$ ). Sin embargo, y a pesar de que registra menor cantidad de días de desconfort, el índice P considera una variable que predomina en el área de estudio: el viento. Al incluirla en el cálculo puede definir mejor la sensación de confort percibida por la población. De este modo, el índice P sería el más recomendable para su utilización en el balneario de Monte Hermoso. A pesar de las diferencias mencionadas, los índices ubicaron al 2009 como el año que presentó mayor malestar térmico.

\section{CONCLUSIONES}

El continuo crecimiento urbano y poblacional de las zonas costeras hace necesario el conocimiento de sus características bioclimáticas. De este modo, se logra una mejor planificación de los recursos y las actividades, además de asegurar el bienestar de la población. Los parámetros meteorológicos más importantes para el sentir climático del hombre son: temperatura, humedad y viento. Este último 
caracteriza a la costa bonaerense e influye sobre los valores obtenidos de confort climático.

Una de las actividades que mayor crecimiento ha experimentado en los últimos tiempos es la turística. Diversas investigaciones la relacionan con el tiempo y el clima, ya que pueden ser factores limitantes para su desarrollo. En este sentido, el conocimiento y utilización de índices de confort facilitan la planificación y favorecen el desarrollo de las tareas recreativas de la población.

Para el área de estudio, la aplicación de estos tres índices permitió observar las variaciones de los valores de confort de un año a otro. El año 2009 se caracterizó por ser el más agobiante. La diferencia entre los índices se vio representada por una mayor cantidad de días de desconfort para THI y H que para el $\mathrm{P}$, aunque este último es el más representativo al incluir, en su cálculo, la variable viento, tan característica en la zona.

El área de estudio se afianza como destino turístico al registrar, año a año, un incremento en la afluencia de visitantes. Por este motivo, resulta conveniente brindar la información sobre confort climático al municipio de Monte Hermoso para facilitar la planificación de las actividades turísticas. Además, permite prevenir a la población, en especial a aquellos grupos que representan riesgos (niños y ancianos), sobre el desconfort en días con vientos del Norte.

Con el fin de evitar riesgos en la salud de residentes y turistas, el mes de marzo se perfila como óptimo para el desarrollo de actividades recreativas y de esparcimiento. Los meses de enero y febrero son los que registran la mayor cantidad de días con sensación de desconfort. Esta situación requiere una planificación, por parte de la municipalidad, de las actividades que se van a realizar con el objeto de beneficiar a la comunidad.

\section{BIBLIOGRAFÍA}

Batista Tamayo, L. M. y Matos Pupo, F. (2004): «Aptitud climática del destino turístico Jardines del Rey (Cuba). Los tipos de tiempo», en El Clima entre el Mar y la Montaña. Asociación Española de Climatología y Universidad de Cantabria, Serie A, no 4, pp.561-570.

Besancenot, J. P. (1991): Clima y Turismo. Masson S.A. Barcelona. 223 pp.

Brazol, D. (1951): «La temperatura biológica óptima», Meteoros, Año I, nº 1, pp. 99-106.

Brazol, D. (1954): «Bosquejo bioclimático de la República Argentina», en Meteoros, Año IV, no 4, pp. 381-394.

Capelli de Steffens, A.; Piccolo, M. C. y Campo de Ferreras, A. M. (2005): Clima urbano de Bahía Blanca. Editorial Dunken, Universidad Nacional del Sur, Bahía Blanca, 199 pp. 
De Freitas, C. R. (2001): «Theory, concepts and methods in climate tourist research», en Proceedings of the First International Worshop on Climate, Tourism and Recreation, International Society of Biometeorology, Comission on Climate, Tourism and Recreation, pp. 3-20.

Del Pozo, O. (2001): «El proceso de urbanización y la degradación ambiental del ecosistema costero, Municipio de Monte Hermoso, Provincia de Buenos Aires». Tesis de Magister en Gestión Ambiental del Desarrollo Urbano (inédita). Universidad Nacional del Sur, Bahía Blanca. 249 pp.

Ercolani, P. y Visciarelli, S. (2003): «Características de la demanda turística en el Partido de Monte Hermoso», en II Jornadas Interdisciplinarias del Suroeste Bonaerense, Universidad Nacional del Sur, Bahía Blanca, Argentina, pp. 563-576.

Fernández, E., Marcos, A., Caló, J. y Aldacour, H. (2006): «Balance sedimentario, parámetros meteorológicos y oceanográficos en un sector de la playa de Monte Hermoso, Provincia de Buenos Aires», en: GEOACTA 31, pp. 11-22.

Gómez Martín, M. B., López Palomeque, F. y Marín Vide, J. (2002): «Aptitud climática y turismo. Variaciones geográficas y cronológicas de la potencialidad climático-turística del verano en Cataluña», Eria, 59, pp. 333-345.

Gómez Martín, M. B. (2004a): «Percepción de la demanda y métodos de evaluación de la potencialidad turística de los recursos atmosféricos en Cataluña», en Documents d'Anàlisi Geogràfica, no 44, pp. 43-70.

Gómez Martín, M. B. (2004b): «An evaluation of the tourist potential of the climate in Catalonia (Spain): A regional study», Geografiska Annaler, Series A., no 86 A (3), pp. 249-264.

Hoffmann, J. A. y Medina, L. (1971): «Ensayo de una clasificación bioclimática en la República Argentina», Meteorológica, Volumen II, n 1, 2 y 3, pp. $150-170$.

Höppe, P. (2002): «Different aspects of assessing indoor and outdoor thermal comfort», Energy and Buildings 34, pp. 661-665.

Huamantinco Cisneros, M. A. y Piccolo, M. C. (2008): «El confort estival de Monte Hermoso», en V Jornadas Interdisciplinarias del Sudoeste Bonaerense, Universidad Nacional del Sur, Bahía Blanca, Buenos Aires, Argentina. (En prensa).

INDEC (Instituto Nacional de Estadística y Censos), 2009. Censo Nacional de Población, Hogares y Viviendas del año 2001. [En línea]. Disponible desde Internet en: <http://www.indec.gov.ar/censo2001s2_2/ampliada_index. asp?mode=07>, Julio de 2009. Argentina.

Marini, M. F. y Piccolo, M. C. (2000): «El confort estival en diversos estuarios del Sudoeste Bonaerense», en Actas de las III Jornadas Nacionales de Geografía Física, Universidad Católica de Santa Fe, pp. 169-176. 
Matzarakis, A. (2006): «Weather-and climate-related information for tourism», Tourism and Hospitality Planning \& Development, Volumen 3, n 2, pp. 99-115.

MAYER H. y Höppe P. (1987): «Thermal comfort of man in different urban environments», Theoretical and Applied Climatology 38, pp. 43-49.

Meteorological Service of Canada. (2009). Humidity. [En línea]. Disponible desde Internet en: <http://www.msc-smc.ec.gc.ca/cd/brochures/humidity_e. cfm>, Julio de 2009.

Nikolopoulou, M., Baker, N. y Steemers, K. (2001): «Thermal comfort in outdoor urban spaces: understanding the human parameter», Solar Energy, vol. 7, $\mathrm{n}^{\circ}$ 3, pp. 227-235.

NiKolopoulou, M., Lykoudis S. y KikiRA M. (2003): «Thermal comfort in outdoor spaces: field studies in Greece», Proceedings of the Fifth Internacional Conference on Urban Climate, 4 pp.

Secretaría de Turismo Municipalidad de Monte Hermoso, 2009.

Stathopoulos, T., WU, H. y Zacharias, J. (2004): «Outdoor human comfort in a urban climate», Building and Environment 39, pp. 297-305.

Varela, P. A. (1982): «Un estudio de la brisa de mar en Monte Hermoso e Ingeniero White», en Geofísica, Instituto Panamericano de Geografía e Historia, $\mathrm{n}^{\circ} 17$, pp. 73-85.

Vaquero, M. del C. y Pascale, J. C. (2003): «La definición del perfil turístico a través de la aplicación de un modelo de planificación participativa Plan de Desarrollo Turístico del Partido de Monte Hermoso», en II Jornadas Interdisciplinarias del Suroeste Bonaerense, Universidad Nacional del Sur, Bahía Blanca, Argentina, pp. 613-623.

Vaquero, M. del C., Rordríguez, C. y Trellini, M. (2007): «El turismo residenciado en Monte Hermoso», en Ambiente natural, campo y ciudad, estrategias de uso y conservación en el sudoeste bonaerense: Actas de las IV Jornadas interdisciplinarias del sudoeste bonaerense, Universidad Nacional del Sur, pp. 201-206. 